\title{
Some Consequences of a Universal Tension Arising from Dark Energy for Structures from Atomic Nuclei to Galaxy Clusters
}

\author{
C Sivaram ${ }^{1}$, Kenath Arun ${ }^{2, *}$ and Kiren $\mathrm{OV}^{3}$ \\ ${ }^{1}$ Indian Institute of Astrophysics, Bangalore, 560 034, India \\ ${ }^{2}$ Christ Junior College, Bangalore, 560 029, India \\ ${ }^{3}$ Christ Junior College, Bangalore, 560 029, India
}

\begin{abstract}
In recent work, a new cosmological paradigm implied a mass-radius relation, suggesting a universal tension related to the background dark energy (cosmological constant), leading to an energy per unit area that holds for structures from atomic nuclei to clusters of galaxies. Here we explore some of the consequences that arise from such a universal tension.
\end{abstract}

Keywords: Universal Tension, Dark Energy, Atomic Nuclei, Galaxies, Galaxy Clusters.

\section{INTRODUCTION}

In recent papers [1-5] a new kind of cosmological paradigm was invoked wherein the requirement that for a hierarchy of large scale structures, like galaxies, galaxy clusters, super-clusters, etc. their gravitational (binding) self energy density must at least equal or exceed the background repulsive dark energy density (a cosmological constant as current observations strongly suggests) implies a mass-radius relation of the type:

$\frac{M}{R^{2}}=\frac{c^{2}}{G} \sqrt{\Lambda}$

(i.e. $\frac{G M^{2}}{8 \pi R^{4}}=\frac{\Lambda c^{4}}{8 \pi G}$ gives rise to a universal tension, $\left.T=\frac{M c^{2}}{R^{2}}=\frac{c^{4}}{G} \sqrt{\Lambda}\right)$

This is the background curvature $\mathrm{x}$ superstring tension $[1,6]$. Or this can also be inferred as the local mass $\mathrm{x}$ local curvature. (Superstring tension is $\sim \beta c^{4} / G, \Lambda$ is the cosmological dark energy)

This paradigm focuses on the universe's fundamental structures and symmetries and emphasises a new universal parameter underlying systems from the smallest (atomic nuclei) to the largest (clusters of galaxies), encompassing nearly 80 orders of magnitude in mass and nearly 40 orders of magnitude in size [7-9].

*Address correspondence to this author at the Christ Junior College, Bangalore, 560 029, India; Tel: +91-80-4012 9292; Fax: +91-80- 4012 9222;

E-mail: kenath.arun@cjc.christcollege.edu

\section{NUCLEAR TENSION}

The energy per unit area (surface tension) given by above equations, i.e. $T=\frac{M c^{2}}{R^{2}}=\frac{c^{4}}{G} \sqrt{\Lambda}$, has the same numerical value as that in nuclear physics, $[3,6]$ like the surface tension in the nuclear liquid drop model of $\sim 10^{21} \mathrm{ergs} / \mathrm{cm}^{2}$. This has consequences for the nucleus and nuclear matter.

In the nucleus this nuclear surface tension balances the Coulomb repulsion:

$\frac{Z^{2} e^{2}}{R}=4 \pi R^{2} T$

Where $R=R_{0} A^{1 / 3}, R_{0} \sim 1.5 \times 10^{-13} \mathrm{~cm}$

For $T \sim 10^{21} \mathrm{ergs} / \mathrm{cm}^{2}$, this sets a limit of:

$\frac{Z^{2}}{A}<40$

which agrees with the usual Bohr-Wheeler criterion. The limiting $Z^{2} / A$ against spontaneous fission is given by: [10]

$\frac{Z^{2}}{A} \approx 2\left(\frac{a_{S}}{a_{C}}\right)$

where, $a_{S}=18 \mathrm{MeV}$ and $a_{C}=0.72 \mathrm{MeV}$ are the surface and Coulomb energy factors respectively.

The Bohr-Wheeler condition for fission reduces to:

$\frac{3(Z e)^{2}}{10 R_{0}}>4 \pi R_{0}^{2} T$ 
(With the usual nuclear physics values, $T=u_{0} A^{2 / 3}$, where $u_{0} \approx 10 \mathrm{MeV}$, is the constant in the surface energy contribution $\left(M_{S}\right)$ to the empirical nuclear mass formulas, i.e. $M_{S}=u_{0} A^{2 / 3}$ )

Using the values in the usual data, one finds for stability, the requirement: $\frac{Z^{2}}{A}<39$. For example, an unstable nucleus like U-236, $\frac{Z^{2}}{A} \approx 37$.

The radius of the nucleus is given by, $R=R_{0} A^{1 / 3}, R_{0} \sim 1.5 \times 10^{-13} \mathrm{~cm}$ is a constant. This constant can be related to the cosmological constant, i.e. vacuum energy (DE), which is a very intriguing relation. Consider a wave packet of spread $r$. Its gravitational self energy density is then given as:

$E_{G} \approx \frac{G(\hbar / r c)^{2}}{8 \pi r^{4}}=\frac{G \hbar^{2}}{8 \pi r^{6} c^{2}}$

(from the uncertainty principle, the energy oaf the packet is $\approx \hbar c / r$ )

To be bound by its gravitational attraction this should at least be the same as the repulsive energy density of the cosmological vacuum given by:

$E_{C}=\frac{\Lambda c^{4}}{8 \pi G}$

This gives,

$r^{6}=\frac{L_{P l}^{4}}{\Lambda}$

( $L_{P l}=\sqrt{G \hbar / c^{3}}=1.6 \times 10^{-33} \mathrm{~cm}$ is the Planck's length)

Therefore we have:

$r=\frac{L_{P l}^{2 / 3}}{\Lambda^{1 / 6}}=1.5 \times 10^{-13} \mathrm{~cm}$

This is the value of $r$ used to get the radius of a nucleus of mass number A, i.e. $R=R_{0} A^{1 / 3}$.

So here the constant $R_{0}$ is $\frac{L_{P l}^{2 / 3}}{\Lambda^{1 / 6}}$.

The inverse of $R_{0}$ roughly gives the QCD scale of $\sim 160 \mathrm{MeV}$, i.e., $\Lambda_{Q C D} \approx R_{0} ; \Lambda_{Q C D}^{-1} \approx 160 \mathrm{MeV}$ ! So it appears the coincidence may go beyond the liquid drop model.
As an example, for a nucleus of mass number $\mathrm{A}$ and radius $\mathrm{R}$, binding surface energy can be written as $4 \pi R^{2}\left(A^{2 / 3}-1\right) T$.

For helium nucleus $\mathrm{A}=4$, so $A^{2 / 3} \approx 2.5$. Thus $\Delta E_{H e}=6 \pi r^{2} T$. Substituting the universal value of $T=\frac{\sqrt{\Lambda}}{G} c^{4}$, gives for the binding energy of the helium nucleus

$4 \pi R_{0}^{2}\left(A^{2 / 3}-1\right) \frac{c^{4}}{G} \sqrt{\Lambda}=4.5 \times 10^{-5}$ ergs

Which is precisely the experimental value as in the conversion of $\mathrm{H}$ to $\mathrm{He}$ (to within one percent).

Indeed we have the interesting relation, a dimensionless quantity, connecting gravity, the cosmic vacuum energy and nuclear physics!

$4 \pi R_{0} \frac{c^{3}}{\hbar G} \sqrt{\Lambda} \approx 1$ have:

Considering also the rotation (spin) of the nucleus we

$\frac{Z^{2} e^{2}}{R}+\frac{1}{5} M \omega^{2} R^{2}=4 \pi R^{2} T$

Where the terms on the left are repulsive in nature and that on the right is attractive.

This gives the radius of the size of the nucleus as (where $M=m_{P} A, m_{P}$ is the proton mass):

$R=\left(\frac{Z^{2} e^{2}}{4 \pi T-\frac{1}{5} m_{P} A \omega^{2}}\right)^{1 / 3}$

The angular frequency dependence on nuclear size is given in Fig. (1)

For the limiting case as $R \rightarrow \infty$ :

$4 \pi T=\frac{1}{5} m_{P} A \omega^{2}$

This sets a limit on the frequency as:

$\omega=\left(\frac{20 \pi T}{A m_{P}}\right)^{1 / 2}$

For $A=1$ we have:

$\omega \leq 2 \times 10^{23} s^{-1}$

And the corresponding time period of:

$\tau=\frac{2 \pi}{\omega} \sim 3 \times 10^{-23} s$ 


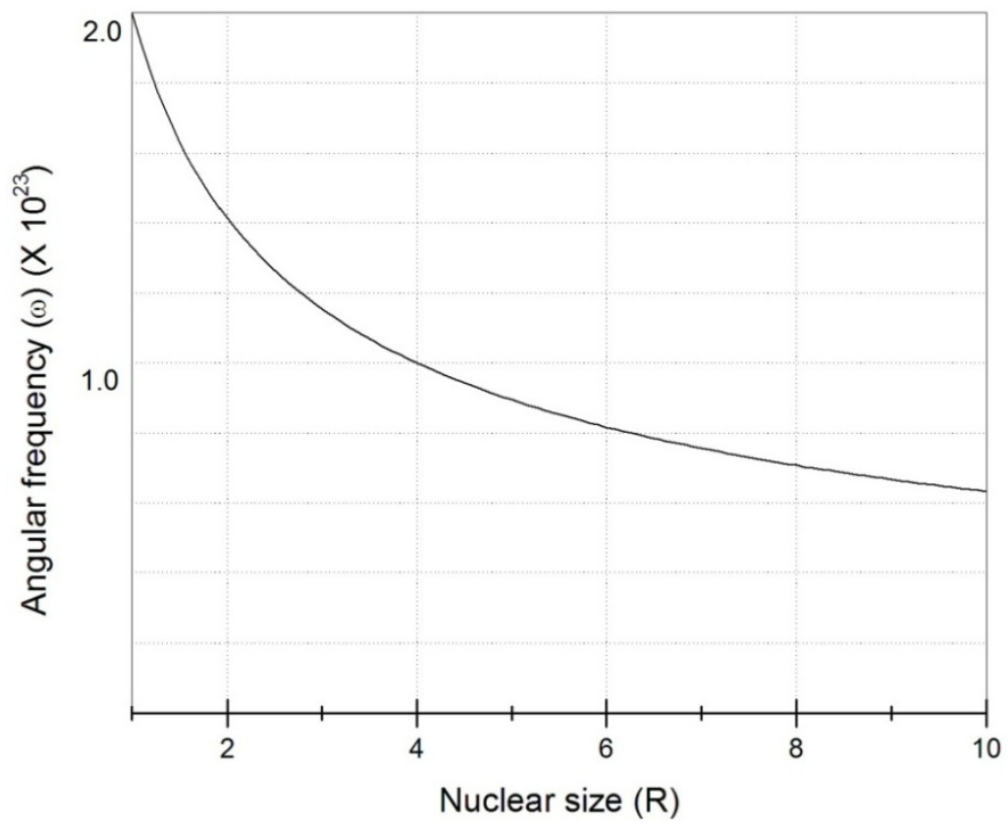

Fig. (1). Dependence of Angular Frequency with Nuclear size.

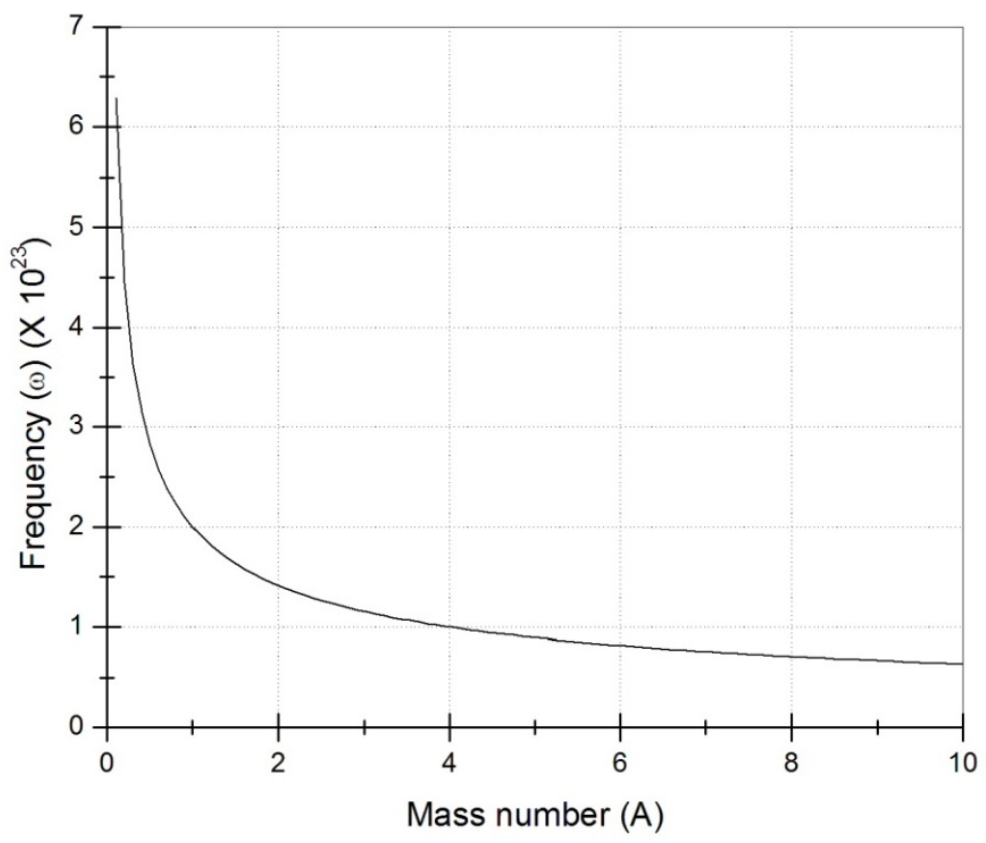

Fig. (2). Variation of angular frequency with A.

This corresponds to the nuclear time scale. Fig. (2) gives the variation of angular frequency with the number of nucleons.

This limit on the frequency will also put a constraint on the rotational energy levels of nucleus:

$\frac{2}{5} M \omega^{2} R^{3}=n \hbar$

$n^{2} \leq 10 A^{7 / 3}$

For $A=10$, we have $n \approx 100$
These states correspond to the yrast states, which are the lowest excited level at high angular momentum $(\sim 70 \hbar)$ as suggested in the following reference [11]. Later observations indicate high angular momentum $(\sim 100 \hbar)$.

Fig. (3) gives the almost linear dependence of the order $(n)$ with the mass number.

\section{GRAVITATIONALLY BOUND STRUCTURE, AN-} GULAR MOMENTUM AND DARK ENERGY

In the case of large, gravitationally bound structures such as galaxies, galaxy clusters, etc. the requirement is that 


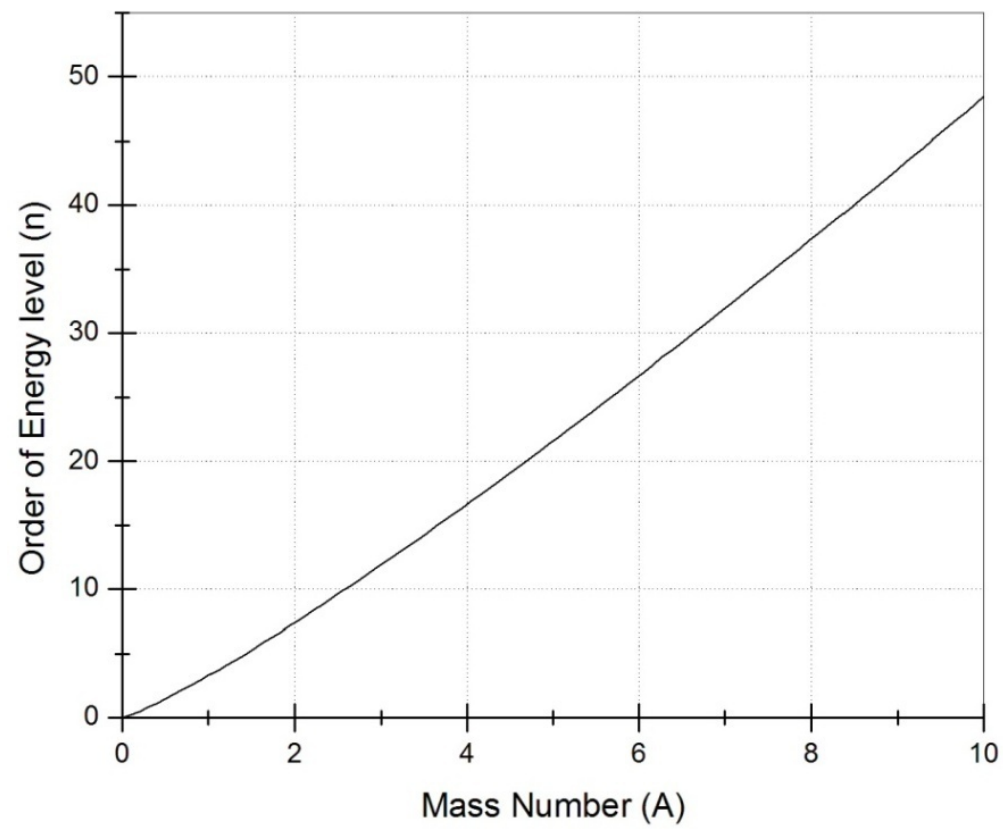

Fig. (3). Variation of $n$ with Mass number.

gravitational self energy density should be comparable to the background cosmic vacuum energy density for the object to be an autonomous structure. That is:

$\frac{G M^{2}}{8 \pi R^{4}}=\frac{\Lambda c^{4}}{8 \pi G}$

This would also give the same result as equation (1), i.e.: $\frac{M}{R^{2}}=\frac{c^{2}}{G} \sqrt{\Lambda}$

Where $\Lambda$ is the cosmological constant with an observed value of $10^{-56} \mathrm{~cm}^{-2}$. This equation holds for a whole range of large scale structures, including the Hubble volume [3].

The $\frac{M}{R^{2}}$ relation is suggestive of a surface tension which has the same universal value for all the large scale cosmic structures from globular clusters, large molecular clouds, all the way to the Hubble universe [1,3]. A kind of universal surface tension, suggesting the holographic picture! [5] (It also holds for the electron!)

The universality of this surface tension again constrains the size of a neutron star. For a neutron star composed on ' $N$ ' neutrons:

$\left(4 \pi R_{N S}^{2} T\right) N=\frac{G M_{N S}^{2}}{R_{N S}}$

For a 2 solar mass neutron star, $N \approx 5 \times 10^{57}$, which matches the observations for the heaviest detected neutron star till date [12]. have:

Considering also the rotation of the neutron stars we $\left(4 \pi R_{N S}^{2} T\right) N=\frac{G M_{N S}^{2}}{R_{N S}}-M_{N S} \omega^{2} R_{N S}^{2}$
This sets a limit on the rotational frequency and the corresponding time period of the neutron star as:

$\omega \approx 10^{4} \mathrm{~s}^{-1}, \tau \approx 0.5 \mathrm{~ms}$

This is consistent with the observations of the millisecond pulsar having the fastest rotational period detected so far, which is $\sim 1.3 \mathrm{~ms}$ [13].

In the case of galaxies, this surface tension balancing the rotational energy can possibly explain the flat rotation curve of the galaxies. That is, for galaxies, their rotation balances this surface tension. This gives:

$4 \pi R_{g a l}^{2} T=M_{g a l} \omega^{2} R_{g a l}^{2}$

Where the rotation frequency and the corresponding time periods are given as:

$\omega=\left(\frac{4 \pi T}{M}\right)^{1 / 2} \approx 3 \times 10^{-12} s^{-1}, \quad \tau \approx 10^{12} s$

Since $M / R^{2}$ is a constant even for a galaxy, the relation given by equation (23) leads to:

$\omega \propto \frac{1}{R_{g a l}}$

$\omega R_{\text {gal }}=$ constant

Therefore, the velocity, which is given by:

$\mathrm{v}=\omega R_{g a l}=\left(\frac{4 \pi T}{M_{g a l} / R_{\text {gal }}^{2}}\right)^{1 / 2}$ 


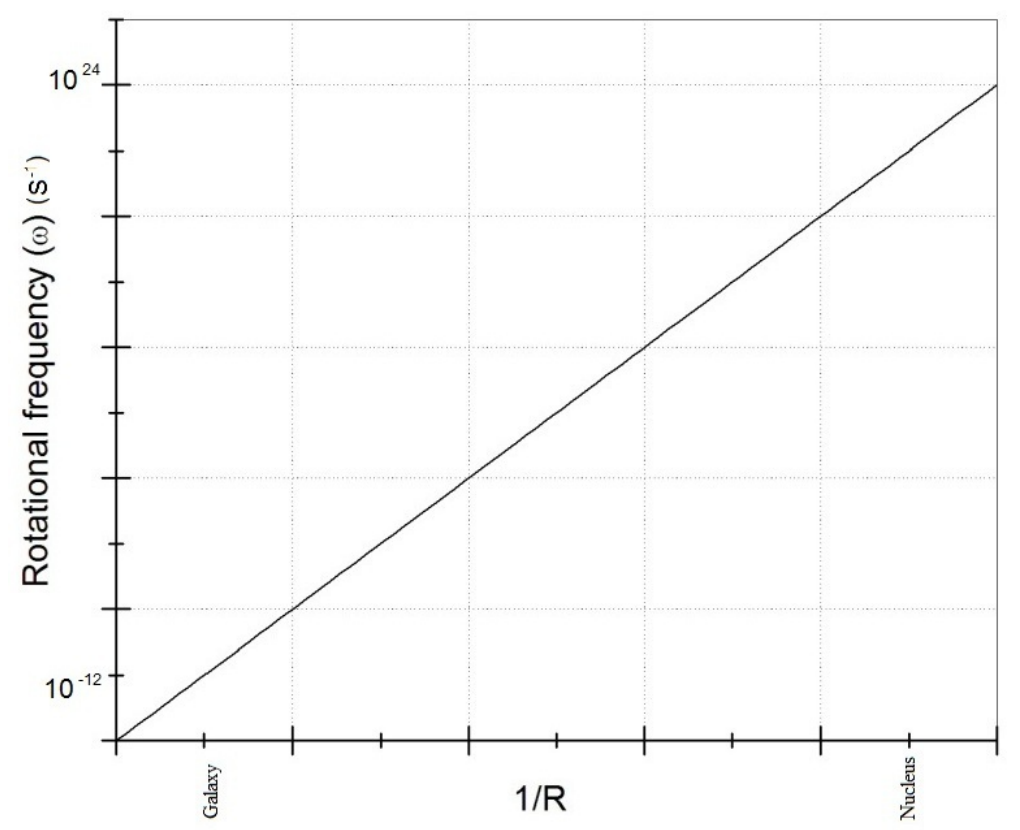

Fig. (4). Variation of rotational frequency with size.

will also be a constant as expected from the galaxy rotation curves! This suggests a velocity independent of radial distance (flat rotation curve) without invoking dark matter.

It is interesting to note that this dependence of rotational frequency going as inverse of the size hold true even right down to the atomic nucleus, as indicated by Fig. (4).

In an earlier work [14] a primordial cosmic rotation was suggested which can give rise to the observed rotation angular momenta of galaxies, galaxy clusters, stellar planetary systems, etc., the origin of which is otherwise not clearly understood.

The angular momentum of the galaxy given by $J_{g a l}=M_{g a l} \omega R_{g a l}^{2}$, is conserved. Therefore we have (again since $M / R^{2}$ is a constant):

$\frac{J_{g a l}}{R_{\text {gal }}^{3}}=\frac{M_{g a l} \omega R_{g a l}^{2}}{R_{\text {gal }}^{3}}=\frac{M_{g a l} \mathrm{v} R_{g a l}}{R_{\text {gal }}^{3}}$ is a constant

This also leads to $\omega R_{g a l}=$ constant and therefore a velocity independent of radial distance.

\section{DYNAMICS OF EVOLVING STRUCTURES}

The requirement that the gravitational self energy density must at least equal or exceed the background repulsive dark energy density implied a mass-radius relation as given by equation (1), for a hierarchy of large scale structures, like galaxies, galaxy clusters, super-clusters, etc.

This mass-radius relation holds good for nebulae too. Any perturbation to it will lead to its collapse and eventual formation of the star (and possibly planetary system). For a typical star of mass, $M_{\text {star }} \approx 10^{33} \mathrm{~g}$, the condition that
$M / R^{2} \approx 1$ implies that the initial size of the nebula be of the order of $R_{N e b} \sim 3 \times 10^{16} \mathrm{~cm}$.

It is also of interest to note that the same value for the tension (arising as we have seen, from the cosmic dark energy $(\Lambda$ term $)$ ) which we have used for galaxies, galaxy clusters, atomic nuclei, etc. also seems to be relevant for the dimensions of planets and stars.

For example, for a typical planetary mass of $M \sim 10^{28} g$, balancing surface energy and gravitational self energy, i.e.

$4 \pi R^{2} T=\frac{G M^{2}}{R}$

we get the radius, which is given by:

$R=\left(\frac{G M^{2}}{4 \pi T}\right)^{1 / 3}$

For $M \sim 10^{28} \mathrm{~g}$, we get $R \approx 5000 \mathrm{~km}$ (the earth radius). The above equation also gives a Jupiter radius of $\sim 10^{5} \mathrm{~km}$ for the corresponding mass.

For a typical stellar mass of $M \sim 10^{33} g$, the above equation implies $R \sim 10^{12} \mathrm{~cm}$. So the range of stellar and planetary sizes is also given by the same value of $T$ ! This suggests a deep underlying connection between the background dark energy ( $\Lambda$-term, which gives the background curvature) and all the structures embedded in this background.

For the large structures we had balance of gravitational energy densities with the background dark energy density. For the planetary and stellar objects, the balance was with surface energies and gravitational self energies. 


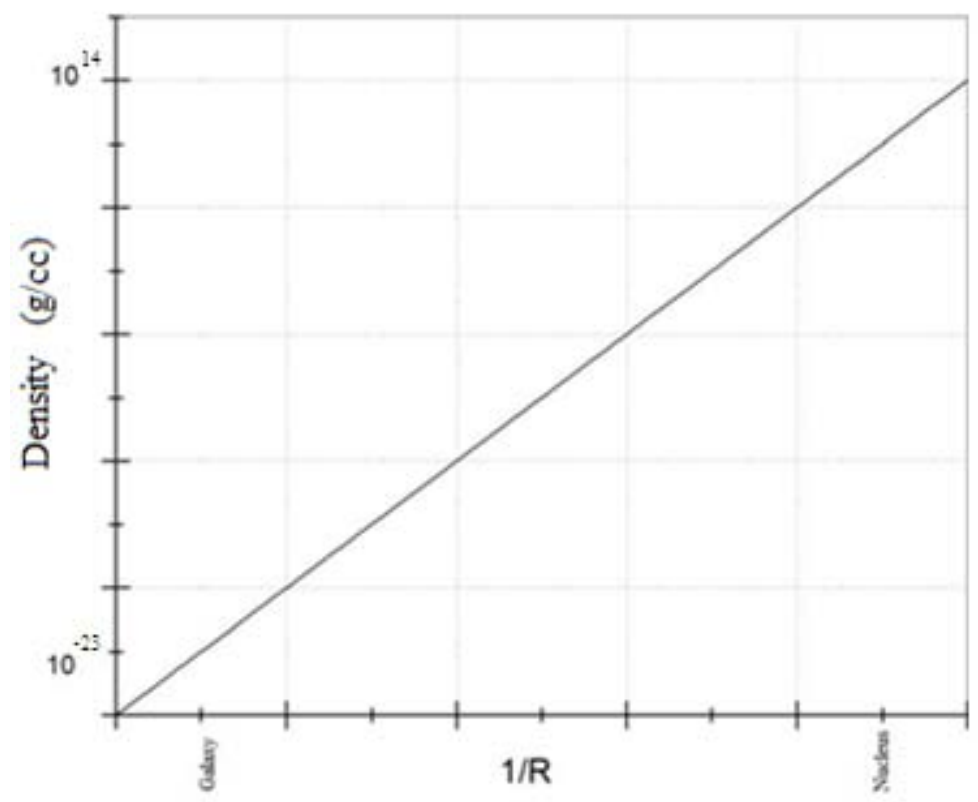

Fig. (5). Variation of density with size.

\section{DENSITIES OF VARIOUS STRUCTURES}

As noted above, we had a universal, $M / R^{2}$ ratio, i.e. a ubiquitous surface tension of $\sqrt{\Lambda} c^{4} / G \approx 10^{21} \mathrm{ergs} / \mathrm{cm}^{2}$, underlying all entities from nuclei to galaxy superclusters! But we know that nuclear density is $\sim 10^{14} \mathrm{~g} / \mathrm{cc}$, superclusters have a density of $\sim 10^{-25} \mathrm{~g} / \mathrm{cc}$. How to understand this diversity in densities?

It is just that the average density is $\sim \frac{M}{R^{3}}$, so that if we have the universal $T=\frac{M}{R^{2}}=\frac{c^{2}}{G} \sqrt{\Lambda} \sim 1 \mathrm{~g} / \mathrm{cm}^{2}$, the densities of the various structures considered would scale as $\frac{M}{R^{3}}$, i.e. $\rho \propto \frac{T}{R} !\left(\right.$ As $T=M / R^{2}$, so $M / R^{3}$ is just $\left.T / R\right)$

As $T$ is a universal constant the density simply scales as $1 / R$. (In connection with surface tension, this is the Laplace pressure for a droplet). Thus for a nucleus $R \sim 1$ fermi, we have $\rho \sim 10^{13}-10^{14} \mathrm{~g} / \mathrm{cc}$.

For a galaxy $R \sim 10^{23} \mathrm{~cm}$, we have $\rho \sim 10^{-23} \mathrm{~g} / \mathrm{cc}$. For a super-cluster $R \sim 10^{25} \mathrm{~cm}, \rho \sim 10^{-25} \mathrm{~g} / \mathrm{cc}$. And for the Hubble volume, $\rho \sim \frac{1}{R_{H}} \sim \frac{1}{\sqrt{\Lambda}} \sim 10^{-29} \mathrm{~g} / c c$, just what is observed!

So we have another universal result: $\rho R=$ constant

Holding from nuclei to the universe, as shown in Fig. (5).

\section{NUCLEAR VIBRATIONAL AND ROTATIONAL ENERGY LEVELS}

In connection with the energy levels of the nucleus, including both vibrational and rotational levels, we can invoke the liquid drop model of nuclei. In the drop model there is equilibrium between surface tension and Coulomb repulsion. Small perturbations of the drop surface of radius R by $\delta(r)$ gives changes in surface energy (surface given by $F(r, \theta, \phi)=$ constant $)$, which can be expanded in spherical harmonics (like in fluid mechanics of incompressible liquid spheres).

Thus:

$\delta=R \sum_{l, m} C_{l, m} Y_{l, m}$

$L^{2} Y_{l, m}=l(l+1) Y_{l, m}$

The surface energy is perturbed as:

$\delta E_{S}=\frac{T R^{2}}{2} \sum_{l, m}(l-1)(l+2) C_{l, m} C_{l, m}^{*}$

While the electrostatic (repulsive) Coulomb energy is perturbed as:

$\delta E_{C}=-\frac{3 Z^{2} e^{2}(l-1)}{4 \pi(2 l+1)} C_{l, m} C_{l, m}^{*}$ 
Finally we can write the Hamiltonian including also the kinetic energy:

$$
\sum_{l, m} \frac{\rho R^{5} \omega^{2}}{2 l} C_{l, m} C_{l, m}^{*}
$$

The lowest mode being $l=2$ we have the energy levels of a five-dimensional harmonic oscillator as:

$$
E=\hbar \omega_{l}(n+5 / 2)
$$

This for $l=2$ gives the ground state energy level as:

$$
E_{0}=\frac{5}{2} \hbar\left(\frac{8 \pi}{3 A m_{n}}\left(4 T-\frac{3 Z^{2} e^{2}}{10 \pi R^{3}}\right)\right)^{1 / 2}
$$

For a nuclei of $Z=20, A=40$, the above equation gives a ground state energy of:

$$
E_{0} \approx 10^{-5} \text { ergs } \approx 10 \mathrm{MeV}
$$

The higher levels will be in multiples of $10 \mathrm{MeV}$.

For $l=0$, stability is given by:

$$
T<\frac{3 Z^{2} e^{2}}{4 \pi R^{3}}, R=R_{0} A^{1 / 3}
$$

The higher vibrational excited states are given by $n=1,2, \ldots$, etc.

We can include the rotational energy levels (like in atomic spectroscopy). Thus rotational levels are $n \hbar \omega_{r o t}$. The limiting values of $\omega_{\text {rot }}$ for various $A$ have been given above.

Energy levels of rotation are:

$$
E_{r o t}=\frac{\hbar^{2}}{2 I} l(l+1)
$$

Where the moment of inertia of the nuclei is: $I=\frac{2}{5} M R^{2}$

The rotational energy is then given as:

$E_{\text {rot }} \approx 0.1 \mathrm{MeV}$

And the total energy is:

$$
E_{\text {total }}=E_{v i b}+E_{r o t}
$$

For various $n, l$, etc.

Similar relations as those above hold also for (nuclei of) primordial galaxies, provided we replace the Coulomb energy term with the gravitational energy. This would also have a negative sign as it is binding energy.

In other words the replacement $\left(\frac{Z e}{R^{3}}\right)^{2}$ by $G \rho^{2}$ would give the result. That is, the surface energy is perturbed as:

$$
\delta E_{S}=\frac{T R^{2}}{2} \sum_{l, m}(l-1)(l+2) C_{l, m} C_{l, m}^{*}
$$

And the gravitational energy is perturbed as: [15]

$\delta E_{G}=-\frac{3 G \rho^{2} R^{6}(l-1)}{4 \pi(2 l+1)} C_{l, m} C_{l, m}^{*}$

The tension $(T)$ term would be the same. Scaling relations are as before and equation (37) will not apply to galaxies! The frequency of oscillation due to the perturbation for the galaxies is given as:

$\omega=\left(\frac{8 \pi}{3}\left(\frac{4 T}{M}-G \rho\right)\right)^{1 / 2}$

For a typical galaxy of $M \approx 10^{44} \mathrm{~g}, \rho \approx 10^{-24} \mathrm{~g} / \mathrm{cc}$, the frequency is $\omega \approx 10^{-11} s^{-1}$.

These oscillations will emit gravitational waves, where the quadrupole gravitational power is given by: [16]

$P_{G W}=\frac{G}{c^{5}} M^{2} R^{4} \omega^{6}$

And for a typical galaxy of $M \approx 10^{44} \mathrm{~g}, R \approx 10^{23} \mathrm{~cm}$, this gives:

$P_{G W} \sim 10^{54}$ ergs $/ s$

The corresponding strain produce on a detector, which is given as $h=\frac{G E_{G W}}{c^{4} r} \sim 10^{-20}$, which is within the limits of proposed space based gravitational wave observatories like LISA. The sensitivity limits of proposed space based gravitational wave detectors, like LISA, OMEGA and DECIGO, are expected to be within $10^{-23}-10^{-24}$. This is well within the limits calculated by the theoretical model here $[17,18]$.

\section{CONCLUSION}

In this paper, we have extended our earlier work (which gave rise to a mass-radius relation) with a universal value of a surface tension $\left(\sim 10^{21} \mathrm{ergs} / \mathrm{cm}^{2}\right)$ arising from the requirement that the binding energy density of gravitationally bound objects be at least equal or exceed the background repulsive dark energy density. This universal tension arising from dark energy dominating three-fourths of the universe, leads to various consequences for a hierarchy of objects, from atomic nuclei to galaxy clusters. This can for instance set a limit on the rotational energy levels of a nucleus; set the dimensions of planets and stars; to even explain the flat rotation curve of galaxies without invoking dark matter and limit the size of galaxy clusters. In short, we have a new paradigm encompassing features of structures ranging over eighty orders in mass and forty orders in length scale.

\section{CONFLICT OF INTEREST}

The author confirms that this article content has no conflicts of interest. 


\section{ACKNOWLEDGEMENTS}

Declared none.

\section{REFERENCE}

[1] Sivaram C. Some aspects of MOND and its consequences for cosmology. Astrophys Space Sci 1994; 215: 185.

[2] Sivaram C. MOND, dark matter and the cosmological constant. Astrophys Space Sci 1994; 219: 135.

[3] Sivaram C. Scaling Relations for self-Similar Structures and the Cosmological Constant. 8 January 2008: Available from: http://arxiv.org/abs/0801.1218.

[4] Sivaram C, Arun K. On a minimum Column Density for Massive Star Formation. 13 February 2012: Available from: http://arxivweb3.library.cornell.edu/abs/1205.4623v1.

[5] Sivaram C, Arun K. Holography, dark energy and entropy of large cosmic structures. Astrophys Space Sci 2013; 348: 217.

[6] Sivaram C. $21^{\text {st }}$ century astrophysics, In: Saha SK, Rastogi VK, Eds. Anita Publications, New Delhi 2005, p.16.

[7] Oldershaw RL. The self-similar cosmological paradigm: A new test and two new predictions. Astrophys J 1987; 322: 34.

[8] Sivaram C. Scale transformations and evolution of the early universe. Astrophys Space Sci 1993; 207: 317.
[9] Sivaram, C. Some further effects of discrete scale transformations. Astrophys Space Sci 2000; 271: 321.

[10] Bohr N, Wheeler JH. The mechanism of nuclear fission. Phys Rev 1939; 56: 426.

[11] Grover JR. Shell-Model calculations of the lowest-energy nuclear excited states of very high angular momentum. Phys Rev 1967; 157: 832.

[12] Crawford F, Robert JWT, Hessels SM, et al. A survey of 56 midlatitude EGRET error boxes for radio pulsars. Astrophys J 2006; 652: 1499 .

[13] Hessels JWT, Ranson SM, Stairs IH, et al. A Radio Pulsar Spinning at $716 \mathrm{~Hz}$. Science 2006; 311: 1901.

[14] Sivaram C, Arun K. primordial rotation of the universe, hydrodynamics, vortices and angular momenta of celestial objects. the Open Astron J 2012; 5: 7.

[15] Lamb H. Hydrodynamics (6 ${ }^{\text {th }}$ ed). Dover: New York 1945.

[16] Sivaram C, Arun K. Thermal gravitational waves from primordial black holes. The Open Astron J 2011; 4 (Suppl 1-M3): 72.

[17] Stebbins R. Rightsizing LISA. 6 April 2009: Available from: http://arxiv.org/abs/0904.1029.

[18] Kawamura S, Ando M, Nakamura T, et al. The Japanese space gravitational wave antenna - DECIGO. J. Phy: Conf Ser 2008; 122: 012006

Received: September 08, 2013

(C) Sivaram et al.; Licensee Bentham Open.

This is an open access article licensed under the terms of the Creative Commons Attribution Non-Commercial License (http://creativecommons.org/licenses/by-nc/3.0/) which permits unrestricted, non-commercial use, distribution and reproduction in any medium, provided the work is properly cited. 